\title{
Approches participatives : une solution pour le suivi des pêcheries récifales?
}

\author{
Ambroise Brenier ${ }^{1}$, Jamal Mahafina ${ }^{2}$, René Galzin ${ }^{3}$, Jocelyne Ferraris ${ }^{4}$ \\ 1 Océanologue, IRD/EPHE, Université de Perpignan, 66860 Perpignan cedex, France \\ 2 Biologiste, IRD, Institut halieutique et des sciences marines, Toliara 601, Madagascar \\ 3 Ichtyologue, CNRS-EPHE, USR 3278, Laboratoire d'excellence Corail, Université de Perpignan, 66860 Perpignan cedex, France \\ 4 Biostatisticienne, IRD, UR227, Laboratoire d'excellence Corail, Observatoire océanologique de Banyuls, 66650 Banyuls-sur-Mer, \\ France
}

Cet article explore un aspect de la relation entre sciences et sociétés que la communauté francophone, à la différence des Anglo-Saxons ainsi que l'indique la bibliographie mobilisée, analyse moins communément : l'intégration d'amateurs dans la collecte de données. Ce que l'on désigne par « suivi participatif » renvoie aux problèmes que soulèvent l'enrôlement et l'intéressement de la population locale dans des dispositifs de monitoring environnemental au travers de la quête d'informations, aussi bien qualitatives que quantitatives. Dans un contexte de faible conscience écologique comme c'est le cas ici, un tel suivi a son intérêt mais aussi ses limites. Dans tous les cas, il suppose que la recherche se donne les moyens de son encadrement, pariant en quelque sorte sur son efficience pédagogique et même politique.

La Rédaction

\section{Mots-clés :}

mer ; ressources naturelles; suivi participatif ; pêcheries récifales ; Indo-Pacifique

\begin{abstract}
Résumé - La gestion durable des écosystèmes coralliens nécessite de disposer d'informations régulières concernant l'impact des pêcheries sur les ressources récifales et sur les écosystèmes dont elles dépendent. Les suivis intégrés des ressources, des habitats et des espèces associées qui visent à fournir ce type d'informations sont difficiles à mettre en œuvre, car ils impliquent de nombreuses ressources humaines, techniques et financières. Face à ces problèmes, la participation de la société civile dans les programmes de suivi semble en mesure d'apporter des solutions. Dans le but de tester cette hypothèse, des méthodes de suivi participatif ont été mises en œuvre afin de caractériser des pêcheries récifales sur trois socioécosystèmes contrastés : l'île de Moorea et l'atoll de Tikehau en Polynésie française, et le littoral de Tuléar à Madagascar. Les résultats de cette étude soulignent la pertinence de certains suivis participatifs pour fournir une vision écosystémique dans le cas de collecte de données ponctuelles et de large envergure, et leur intérêt moindre pour la collecte continue de données sur de longues échelles de temps. Dans tous les cas, une structure de coordination et l'implication de scientifiques s'avèrent primordiales pour assurer pérennité et fiabilité au système de suivi.
\end{abstract}

\begin{abstract}
Participatory approaches and reef fisheries monitoring. In order to ensure a sustainable management of reef fisheries, data need to be collected regularly on the effect of these fisheries on both fish resources and the ecosystems that sustain them. Habitat and resources monitoring programs can provide such information, but are difficult to implement as they involve a range of technical, financial and human resources. Regarding these problems, the involvement of the local population in monitoring programs, called participatory monitoring, seems to offer solutions. Indeed, participatory monitoring has the potential of increasing the number of data collected at low cost, while taking advantage of the traditional ecological knowledge available locally. Some questions need clarifying, however: which methods can bring relevant information for the monitoring of reef fisheries while being appropriate to the motivations and skills of the local population? Is the degree of participation of the local population sufficient to make the monitoring program work, particularly over the long term? To answer these questions, participatory monitoring methods commonly used for coral reef ecosystems have been implemented to assess reef
\end{abstract}

Auteur correspondant : A. Brenier, ambroise.brenier@gmail.com 
fisheries at three different sites: Moorea island and Tikehau atoll in French Polynesia, and the Bay of Tulear in Madagascar. While highlighting the relevance of some participatory monitoring programs for snapshot and large-scale surveys, the results of this study also show their lesser interest for regular data gathering on long-time scales. In any case, strong coordination and supervision by scientists are considered essential.

\section{Pertinence des suivis participatifs en milieu corallien}

Les pêcheries récifales exploitent les ressources des écosystèmes coralliens qui abritent une biodiversité tout aussi importante que celle des forêts tropicales (Connell, 1978). Elles sont caractérisées par une grande diversité de stratégies d'exploitation (multispécifiques et multiengins) qui s'expliquent autant par la richesse du milieu exploité que par l'adaptation des populations locales pour exploiter ces écosystèmes (Ferraris et Cayré, 2003). D'une importance capitale pour le développement économique et la sécurité alimentaire de nombreux pays en développement (Dalzell et al., 1996), les pêcheries récifales font l'objet d'une attention particulière à l'échelle mondiale face à la dégradation des récifs coralliens (Wilkinson, 2004). L'exploitation intensive de ces ressources est l'une des causes principales de dégradation des écosystèmes coralliens (Jackson et al., 2001) en raison de l'impact sur les espèces ciblées et des dégâts occasionnés sur les communautés associées et leurs habitats, et donc sur les structures et fonctions de l'écosystème (Mumby et al., 2006). Assurer la durabilité biologique des pêcheries nécessite que les espèces prélevées et les méthodes d'exploitation ne mettent pas en péril les capacités des ressources à se régénérer et des écosystèmes à fournir les services qui supportent ces ressources (Hilborn, 2007). C'est cette approche que préconise la gestion écosystémique des pêches (FAO, 2003 ; Pikitch et al., 2004) qui vise à mieux intégrer la santé des écosystèmes, les besoins de la société (sécurité alimentaire, emplois) et les objectifs de gestion en matière de conservation et d'exploitation des ressources. L'implication de la société civile est un des principes fondamentaux de la gestion écosystémique (Garcia et al., 2003) et le cadre conceptuel des indicateurs du type pression-impactréponse, une des solutions préconisées pour répondre aux défis de la gestion écosystémique (Caddy, 2004). Ainsi, des indicateurs de pression sur l'écosystème, dont ceux fondés sur les activités de pêche, et des indicateurs de réponse des ressources, biocénoses et biotopes, ainsi que les valeurs de référence associées, sont requis pour favoriser une gestion écosystémique des pêches (Jennings, 2005).

Est appelée ici « suivi écosystémique des pêcheries » la collecte d'informations fournissant un panel d'indicateurs sur l'état des ressources exploitées et des activités de pêche, et sur les changements écologiques, dans le but de favoriser l'évaluation et la prise de décision pour une gestion durable des écosystèmes coralliens. En effet, un seul indicateur ne pouvant résumer la complexité du socioécosystème, l'utilisation conjointe de plusieurs indicateurs décrivant le système pêche est recommandée (Johannes, 1998 ; Clua et al., 2005 ; Trenkel et al., 2007). Les variables biologiques concernent les populations exploitées, leur peuplement et habitats associés ; les variables socioéconomiques portent sur l'activité de pêche, les menaces et la consommation en produits de mer (Tab. 1).

La collecte des données nécessaires au suivi des pêcheries récifales, notamment sur de larges échelles spatiales et temporelles du fait des caractéristiques de ces pêcheries dispersées et multiformes, est difficile et coûteuse pour les gestionnaires qui disposent de budgets et d'effectifs limités (Zeller et al., 2006). La nécessité de disposer d'informations non seulement sur les ressources ciblées, mais également sur les communautés associées et les habitats dont elles dépendent, complique encore l'opération. De plus, lorsque les données sur les écosystèmes coralliens proviennent de programmes de recherche impliquant des suivis à long terme, avec des protocoles scientifiques contraignants, celles-ci ne répondent pas toujours aux besoins des gestionnaires (Sheil, 2001), notamment en ce qui concerne l'identification ou l'évaluation de plans de gestion (environnement, pêche, aires marines protégées). Outre le fait que ces programmes sont généralement définis sans concertation avec les acteurs locaux, une des difficultés concerne les supports de diffusion des résultats, notamment pour les activités de recherche peu adaptées à une audience autre que scientifique, ne facilitant pas leur appropriation par les acteurs et usagers locaux (Danielsen et al., 2005a). Ainsi, ces derniers sont généralement insuffisamment concernés par les activités de suivi alors que leur implication dans le processus de gestion des ressources marines semble capitale pour garantir le succès des mesures de gestion (Walmsley et White, 2003 ; Jackson, 2007).

Lorsque la société civile s'implique dans des activités de suivi, on parle alors de " suivi participatif » (Whitelaw et al., 2003). Les participants peuvent être des membres d'associations, des riverains, des usagers du milieu, des écoliers ou toute personne dont la profession n'a pas pour objectif de réaliser des suivis. La participation de la société civile contribuerait à une meilleure efficacité des programmes de suivi pour plusieurs raisons : augmentation de l'effort d'échantillonnage (Delaney et al., 2008), possibilité de collecter des données fiables à faible coût 
Tableau 1. Indicateurs potentiels de l'impact de la pêche en milieu corallien.

\begin{tabular}{|c|c|c|}
\hline $\begin{array}{l}\text { Catégories } \\
\text { d'indicateurs }\end{array}$ & Indicateurs & $\begin{array}{l}\text { Tendance } \\
\text { potentiellement liée } \\
\text { à une surexploitation }\end{array}$ \\
\hline \multirow{8}{*}{$\begin{array}{l}\text { Populations } \\
\text { exploitées }\end{array}$} & Densité & $\downarrow$ \\
\hline & Biomasse & $\downarrow$ \\
\hline & Taille moyenne & $\downarrow$ \\
\hline & Âge moyen & $\downarrow$ \\
\hline & Sex-ratio mâle/femelle (pour les espèces hermaphrodites protogynes) & $\downarrow$ \\
\hline & Fécondité relative & $\downarrow$ \\
\hline & Richesse spécifique & $\downarrow$ \\
\hline & Distance de l'observateur & $\uparrow$ \\
\hline \multirow[t]{6}{*}{ Peuplement } & Proportion des proies des espèces exploitées & $\uparrow$ \\
\hline & Proportion d'espèces piscivores & $\downarrow$ \\
\hline & Proportion des grands herbivores & $\downarrow$ \\
\hline & Proportion des espèces non commerciales & $\uparrow$ \\
\hline & Proportion des espèces présentant des traits de vie courts et rapides & $\uparrow$ \\
\hline & Niveau trophique moyen & $\downarrow$ \\
\hline \multirow[t]{3}{*}{ Habitat } & Couverture corallienne & $\downarrow$ \\
\hline & Couverture des herbiers & $\downarrow$ \\
\hline & Couverture algale & $\uparrow$ \\
\hline \multirow{14}{*}{$\begin{array}{l}\text { Activité } \\
\text { de pêche }\end{array}$} & Captures par unité d'effort (CPUE) & $\downarrow$ \\
\hline & Poids total des captures & $\downarrow$ \\
\hline & Poids des captures d'espèces à haute valeur commerciale & $\downarrow$ \\
\hline & Rendement de la pêche & $\downarrow$ \\
\hline & Proportion des espèces à haute valeur commerciale dans les captures & $\downarrow$ \\
\hline & Taille moyenne de chaque espèce dans les captures & $\downarrow$ \\
\hline & Taille moyenne des captures & $\downarrow$ \\
\hline & Proportion des espèces piscivores dans les captures & $\downarrow$ \\
\hline & Proportion des espèces de grands herbivores dans les captures & $\downarrow$ \\
\hline & Niveau trophique moyen des captures & $\downarrow$ \\
\hline & $\begin{array}{l}\text { Proportion des espèces présentant des traits de vie courts et rapides dans les } \\
\text { captures }\end{array}$ & $\uparrow$ \\
\hline & Distance moyenne des sites de pêche de la zone d'embarquement & $\uparrow$ \\
\hline & Durée moyenne des sorties de pêche & $\uparrow$ \\
\hline & Quantité de poissons consommés & $\downarrow$ \\
\hline \multirow[t]{3}{*}{$\begin{array}{l}\text { Consommation } \\
\text { en poisson }\end{array}$} & $\begin{array}{l}\text { Proportion des espèces à haute valeur marchande dans la part des captures } \\
\text { destinées à l'autoconsommation }\end{array}$ & $\downarrow$ \\
\hline & Proportion des captures destinées à l'autoconsommation & $\downarrow$ \\
\hline & Nombre d'habitants & $\uparrow$ \\
\hline \multirow[t]{5}{*}{ Menaces } & Nombre de pêcheurs & $\uparrow$ \\
\hline & Nombre de bateaux de pêche & $\uparrow$ \\
\hline & Nombre d'engins de pêche & $\uparrow$ \\
\hline & Nombre ou proportion des engins de pêche destructeurs & $\uparrow$ \\
\hline & Intensité et distribution de l'effort de pêche & $\uparrow$ \\
\hline
\end{tabular}

Liste dressée à partir d'une compilation d'articles portant sur l'impact des pêcheries récifales et sur les indicateurs de suivi de ces pêcheries (Russ, 1991 ; Jennings et Polunin, 1996 ; Rochet et Trenkel, 2003 ; Clua et al., ). 
(Rist et al.,2010 ; Goffredo et al.,2010), prise en compte des connaissances empiriques du milieu, notamment les perceptions sur l'évolution des ressources, habitats et usages (Wiber et al., 2004 ; Bunce et al., 2008 ; Rochet et al., 2008), et enfin, lorsque les participants sont prêts à payer pour participer aux programmes, possibilité de nouvelles sources de financement pour les projets de conservation (Newman et al., 2003).

Cependant, le suivi participatif présente un défi majeur : celui de proposer des méthodes de collecte de données adaptées aux connaissances, compétences et motivations des participants, tout en assurant la validité scientifique des données produites et leur pertinence pour le suivi de l'état des ressources et des écosystèmes dont elles dépendent (Stokes et al., 1990).

Les approches participatives sont-elles pertinentes pour contribuer au suivi des pêcheries récifales ${ }^{1}$ ? Nous émettons l'hypothèse que face aux enjeux actuels liés à la perturbation des récifs coralliens, à la gestion halieutique et aux difficultés rencontrées dans la mise en œuvre des programmes de suivi des pêcheries récifales, les suivis participatifs devraient être en mesure d'apporter des informations pertinentes. Afin de tester cette hypothèse, une approche expérimentale a été privilégiée. Les principales méthodes de suivi participatif utilisées en milieu corallien ont été mises en œuvre sur trois sites d'étude. Cette expérience a ensuite été analysée selon trois critères : i) adéquation entre les méthodes, compétences disponibles et besoins en matière de suivi des pêcheries récifales ; ii) degré d'implication et assiduité des participants ; iii) intérêt des informations générées pour l'évaluation des pressions et impacts des pêcheries récifales sur l'écosystème corallien.

Dans le cadre de l'expérimentation, les sites choisis correspondent à trois socio-écosystèmes contrastés : l'île haute de Moorea et l'atoll de Tikehau en Polynésie française et la baie de Tuléar à Madagascar. Les conditions socioéconomiques et culturelles des populations résidentes en Polynésie française et à Madagascar sont très différentes. L'expérience à Tuléar permet d'évaluer la faisabilité et la pertinence des suivis participatifs dans un contexte de pays en voie de développement où se trouve la majorité des pêcheries récifales. En effet, c'est surtout dans ces pays, aux ressources techniques et financières limitées, que le suivi participatif montre tout son intérêt, par sa capacité à fournir de nombreuses données, en utilisant les compétences locales disponibles, à faible coût.

\footnotetext{
1 Cette recherche a pour origine une demande formulée par les gestionnaires de Polynésie française qui s'interrrogeaient sur la possibilité d'impliquer les populations locales dans la collecte de données utiles aux Plans de gestion de l'environnement marin (PGEM). Une partie de la présente recherche a fait l'objet de présentations lors de trois congrès (voir Brenier et al., 2007 ; Brenier et al., 2009a ; Brenier et al., 2009b).
}

Bien que la Polynésie dispose de plus de moyens qu'un pays en développement, elle est cependant confrontée au suivi des écosystèmes coralliens de 76 îles habitées, éparpillées sur un espace aussi vaste que l'Europe. La diversité des sites d'étude transparaît également à travers les stratégies d'exploitation variées qui s'y exercent. À Moorea comme à Tikehau, la pêche de loisir est très présente, alors qu'à Tuléar la pêche piroguière est pratiquée exclusivement par des pêcheurs professionnels. Par ailleurs, à Tuléar, il existe une très importante pratique de pêche à pied contrairement aux sites de Moorea et Tikehau. Enfin, la diversité des trois sites se retrouve au niveau de la taille des communautés riveraines et du degré d'exploitation des ressources récifales, illustrée par le rapport entre le nombre d'habitants et la surface exploitable par la pêcherie récifale, indicateur potentiel du degré d'exploitation de la zone. Tikehau abrite une petite population de quelques centaines $\mathrm{d}$ 'habitants (le nombre $\mathrm{d}^{\prime}$ habitants par $\mathrm{km}^{2}$ de surface exploitable par la pêcherie récifale est proche de 1 ), alors qu'ils sont plus d'une dizaine de milliers à Moorea (environ 300 habitants $/ \mathrm{km}^{2}$ de surface exploitable) et plus d'une centaine de milliers à Tuléar (environ 750 habitants $/ \mathrm{km}^{2}$ de surface exploitable). Les trois sites choisis ont par ailleurs deux caractéristiques communes : la présence $\mathrm{d}^{\prime} u n e$ équipe scientifique sur place, indispensable pour assurer le suivi de l'expérience, et l'habitude d'être régulièrement enquêté par des organismes divers et variés (scientifiques, ONG, étudiants), ce qui constitue une contrainte pour toute étude de sciences humaines et sociales.

\section{Démarche expérimentale}

La démarche utilisée est fondée, d'une part, sur l'inventaire des approches participatives utilisées en milieu corallien et des informations pertinentes pour assurer un suivi écosystémique des pêcheries récifales et, d'autre part, sur l'évaluation de leur mise en œuvre et des résultats obtenus. Les principales approches de suivi participatif des pêcheries et des milieux coralliens décrites dans la littérature scientifique sont présentées dans le tableau 2. Tandis que les principaux indicateurs de suivi de l'activité de pêche et de ses impacts sur les écosystèmes sont détaillés dans le tableau 1 . Les approches de suivi participatif couramment utilisées recouvrent des enquêtes de perception sur l'évolution de l'écosystème, des suivis continus des captures de pêche, des suivis épisodiques in situ de l'état des ressources et de l'écosystème, et des enquêtes auprès des ménages afin de recueillir rapidement des données sur la consommation et l'activité de pêche auprès de grands échantillons. Les enquêtes de perception sont fondées sur des entretiens semi-directifs menés individuellement ou en groupe avec des usagers du milieu marin (pêcheurs, mareyeuses, moniteurs de plongée) volontaires, répondant à deux 
Tableau 2. Méthodes de suivi participatif couramment utilisées en milieu corallien.

\begin{tabular}{|c|c|c|c|c|c|}
\hline Objectif & Méthode & Participants & Avantages & Inconvénients & Références \\
\hline $\begin{array}{l}\text { Obtenir } \\
\text { des statistiques } \\
\text { de pêche } \\
\text { de subsistance }\end{array}$ & $\begin{array}{l}\text { Questionnaires à } \\
\text { l'attention des } \\
\text { ménages avec des } \\
\text { fiches de relevés } \\
\text { pour le suivi, } \\
\text { pendant une } \\
\text { semaine, de la } \\
\text { consommation en } \\
\text { poissons, et des } \\
\text { sorties de pêche }\end{array}$ & $\begin{array}{l}\text { Écoliers âgés de } \\
14 \text { à } 18 \text { ans }\end{array}$ & $\begin{array}{l}\text { Largeséchantillons } \\
\text { couverts en peu de } \\
\text { temps } \\
\text { Nombreux } \\
\text { bénéfices en } \\
\text { matière } \\
\text { d'éducation- } \\
\text { sensibilisation }\end{array}$ & $\begin{array}{l}\text { Échantillon de } \\
\text { ménages non } \\
\text { représentatif de } \\
\text { l'ensemble des } \\
\text { ménages } \\
\text { Faible } \\
\text { proportion de } \\
\text { fiches de relevés } \\
\text { complétées } \\
\text { correctement }\end{array}$ & (Hosch, 2000) \\
\hline $\begin{array}{l}\text { Détecter des } \\
\text { changements } \\
\text { écologiques } \\
\text { dans } \\
\text { l'écosystème } \\
\text { corallien }\end{array}$ & $\begin{array}{l}\text { Comptage visuel } \\
\text { en plongée de } \\
\text { composants clés de } \\
\text { l'écosystème } \\
\text { corallien }\end{array}$ & $\begin{array}{l}\text { Plongeurs loisirs } \\
\text { Pêcheurs } \\
\text { Membres de la } \\
\text { communauté }\end{array}$ & $\begin{array}{l}\text { Des protocoles } \\
\text { standardisés } \\
\text { permettant des } \\
\text { études sur de } \\
\text { larges échelles } \\
\text { existent }\end{array}$ & $\begin{array}{l}\text { Besoin en } \\
\text { formation } \\
\text { important } \\
\text { Suivi } \\
\text { demandeur en } \\
\text { temps et énergie }\end{array}$ & $\begin{array}{l}\text { (DarwalletDulvy,1996; } \\
\text { Hodgson, } 1999 \text {; Obura, } \\
2001 \text {; Tawake et al., } \\
2001 \text {; Obura et al., } 2002 \text {; } \\
\text { Pattengill-Semmens et } \\
\text { Semmens, } 2003 \text {; } \\
\text { Uychiaoco et al., 2005) }\end{array}$ \\
\hline $\begin{array}{l}\text { Détecter des } \\
\text { tendances } \\
\text { historiques }\end{array}$ & $\begin{array}{l}\text { Questionnaire à } \\
\text { l'attention des } \\
\text { usagers des } \\
\text { ressources } \\
\text { concernant leur } \\
\text { perception de } \\
\text { l'évolution des } \\
\text { composants de } \\
\text { l'écosystème et des } \\
\text { activités de pêche }\end{array}$ & $\begin{array}{l}\text { Usagers du } \\
\text { milieu marin (en } \\
\text { particulier les } \\
\text { pêcheurs) qui } \\
\text { transmettent } \\
\text { leurs savoirs } \\
\text { traditionnels à } \\
\text { l'enquêteur }\end{array}$ & $\begin{array}{l}\text { Les études } \\
\text { scientifiques } \\
\text { portant sur de } \\
\text { longues échelles } \\
\text { temporelles sont } \\
\text { très rares } \\
\text { La connaissance } \\
\text { des tendances } \\
\text { passées est cruciale } \\
\text { pour appréhender } \\
\text { le présent }\end{array}$ & $\begin{array}{l}\text { Données } \\
\text { qualitatives } \\
\text { subjectives }\end{array}$ & $\begin{array}{l}\text { (Debrot et Nagelkerken, } \\
2000 \text {; Obura, } 2001 \text {; } \\
\text { Webb et al., } 2004 \text {; Teh } \\
\text { et al., } 2005 \text {; Uychiaoco } \\
\text { et al., } 2005 \text {; Lunn et } \\
\text { Dearden, 2006; Teh et } \\
\text { Sumaila, 2007) }\end{array}$ \\
\hline $\begin{array}{l}\text { Obtenir des } \\
\text { statistiques de } \\
\text { pêche }\end{array}$ & Cahiers de pêche & Pêcheurs & $\begin{array}{l}\text { Informations de } \\
\text { première main sur } \\
\text { les sorties de pêche } \\
\text { et les captures }\end{array}$ & $\begin{array}{l}\text { Objectivité des } \\
\text { participants } \\
\text { Nécessite un } \\
\text { grand nombre } \\
\text { de participants }\end{array}$ & $\begin{array}{l}\text { (Jennings et Polunin, } \\
1995 \text {; Uychiaoco et al., } \\
\text { 2005) }\end{array}$ \\
\hline $\begin{array}{l}\text { Obtenir des } \\
\text { statistiques de } \\
\text { pêche }\end{array}$ & $\begin{array}{l}\text { Suivi des } \\
\text { débarquements }\end{array}$ & Pêcheurs & $\begin{array}{l}\text { Informations de } \\
\text { première main sur } \\
\text { les sorties de pêche } \\
\text { et les captures }\end{array}$ & $\begin{array}{l}\text { Nécessite un } \\
\text { effort continu } \\
\text { relativement } \\
\text { important }\end{array}$ & $\begin{array}{l}\text { (Obura, } 2001 ; \\
\text { Obura et al., 2002) }\end{array}$ \\
\hline
\end{tabular}

critères : fréquence et durée de pratique de leur métier assurant ainsi une grande connaissance de leur environnement de travail. Les personnes ciblées sont interrogées sur les changements au niveau des ressources, habitats, paramètres environnementaux et activités de pêche qu'ils ont pu observer depuis le commencement de leur activité professionnelle (en moyenne une trentaine d'années). Le suivi des captures est réalisé par des pêcheurs qui détaillent chacune de leur sortie au moyen de cahiers de pêche, ou par des vendeurs sur site qui réalisent un échantillonnage des débarquements. Le suivi in situ consiste à mesurer l'abondance des espèces de poissons commerciaux, par comptage visuel en plongée sous-marine (pour les moniteurs de plongée) ou depuis la surface (pour les pêcheurs). La méthode des enquêtes via les écoles fait appel aux scolaires pour recueillir des données au sein de leurs familles grâce à un questionnaire distribué et expliqué en classe, complété à la maison et retourné quelques jours plus tard. Ce questionnaire comprend trois parties ${ }^{2}$ : le recueil des informations générales concernant les activités de pêche et la consommation en poissons du ménage (adresse et taille du ménage, fréquence de consommation en poissons, origine du poisson consommé, nombre de bateaux, nombre de pêcheurs) ; le détail des repas des trois derniers jours : nom, taille et nombre de poissons consommés ; l'activité de pêche d'un ou plusieurs pêcheurs du ménage (sites, technique et fréquence de pêche, espèces ciblées).

\footnotetext{
2 Pour plus de détails sur le questionnaire utilisé, se référer à Brenier, A., 2009. Pertinence des approches participatives pour le suivi écosystémique des pêcheries récifales. Thèse de doctorat, Université Pierre et Marie Curie, Paris.
} 
Ces approches ont été déployées sur chaque site d'étude, entre fin 2005 et début 2007, en suivant certaines recommandations relatives à la mise en œuvre des suivis participatifs : i) usage des noms locaux (d'espèces et de lieux) et des systèmes locaux de mesure de quantités dans les protocoles de collecte de données (Obura et al., 2002) ; ii) organisation de séances de formation à la pratique des méthodes (Stokes et al.1990) ; iii) distribution de matériel d'aide à la collecte lorsque nécessaire (Gouveia et al., 2004) ; iv) contrôle de la qualité des données (Savan et al., 2003) : élimination des questionnaires incorrectement complétés, tests préalables du degré de connaissance des participants en identification de poissons, recoupement des informations issues des enquêtes de perception; $v$ ) établissement de mesures incitatives telles que la valorisation des participants, la mise en jeu de cadeaux, l'établissement de compensations financières, la restitution des résultats des suivis (Andrianandrasana et al., 2005) ; vi) mise en place d'une structure de coordination pour assurer les fonctions indispensables de mise en œuvre et d'animation (communication, formation, centralisation et analyse des données) du processus de suivi participatif (Conrad et Daoust, 2008).

Le degré d'implication et l'assiduité des participants aux programmes de suivi sont ensuite examinés. Pour chaque méthode mise en œuvre, le nombre de participants est comptabilisé. Les taux de retour des questionnaires ou fiches de collecte sont calculés afin de mesurer l'assiduité des participants, particulièrement pour les méthodes testées sur de longues périodes.

Enfin, l'utilité de ces approches participatives à produire des informations pertinentes pour le suivi des pêcheries récifales est évaluée à la lumière des données collectées sur chaque site. Les indicateurs obtenus sont regroupés sous la forme de tableaux de bord et comparés à des valeurs de référence tirées de la littérature scientifique lorsque disponibles. La qualité et l'utilité des connaissances obtenues à travers l'analyse de ces tableaux de bord renseignent sur la capacité des approches participatives testées à contribuer à une évaluation globale des pressions et impacts de l'exploitation sur le milieu.

\section{Mise en œuvre sur le terrain : les résultats}

\section{Adéquation entre les méthodes et les conditions spécifiques liées à chaque site}

La mise en œuvre des enquêtes de perception n'a pas rencontré de problèmes majeurs. Les personnes ciblées se sont prêtées volontiers à ces entretiens, dont la durée était comprise entre une demi-heure et cinq heures. En revanche, certains obstacles doivent être parfois écartés, avant que la personne enquêtée ne fasse partager à un étranger sa connaissance écologique empirique du milieu marin. Le fait de passer par un intermédiaire, membre de leur communauté, reconnu et jouant également le rôle de traducteur si nécessaire, facilite le premier contact et l'explication de la raison et de l'intérêt de l'enquête. Une attention particulière doit également être apportée à la restitution des résultats de l'enquête auprès des personnes enquêtées qui, sans quoi, se lassent vite de répondre aux enquêtes à répétition. Les éléments de connaissance empirique des usagers dans les enquêtes de perception permettent d'obtenir une vision écosystémique de la pêcherie puisqu'ils couvrent à la fois les populations exploitées, les habitats, les activités de pêche et les menaces sur l'écosystème.

Les débarquements sont plus éparpillés dans le temps et dans l'espace en Polynésie française qu'à Tuléar. Sur ces plages malgaches interviennent des mareyeuses, intermédiaires entre le pêcheur et le consommateur, qui centralisent un certain nombre de captures. Ce phénomène est quasiment inexistant à Moorea et à Tikehau. Ainsi, alors que le suivi des débarquements par les mareyeuses semble être pertinent pour le suivi des captures à Tuléar, les cahiers de pêche individuels, remplis par les pêcheurs, sont mieux adaptés pour le suivi des captures en Polynésie française. Les pêcheurs polynésiens ne rencontrent pas de difficulté technique à remplir ces cahiers. Ainsi, quasiment aucune formation n'est nécessaire. Pour ce qui est du suivi des débarquements par les mareyeuses, une forte implication externe est requise, que ce soit pour former les mareyeuses à la pratique d'échantillonnage de pirogues au débarquement ou pour assurer une supervision régulière du travail de suivi de ces mareyeuses. Les indicateurs d'impact potentiel de la pêche renseignés par cette méthode concernent les captures par unité d'effort et par technique, et la composition des captures par technique et par saison.

La méthode du suivi in situ n'a pas été réalisable à Tuléar en raison de l'absence de club de plongée et de l'illettrisme des pêcheurs, par ailleurs peu disponibles. $\mathrm{Au}$ contraire, en Polynésie française certains moniteurs de plongée et pêcheurs, disposant de bonnes connaissances en identification de poissons, ont mis à disposition du suivi leur temps et leurs moyens logistiques (bateau, matériel de plongée). Mais cette méthode s'est avérée complexe à mettre en œuvre. Tout d'abord, elle nécessite un engagement important des participants, car le comptage visuel en plongée est une méthode fastidieuse, exigeant rigueur, technicité, effort physique et disponibilité. De plus, elle requiert un protocole contraignant (variabilité des observations liée à l'observateur et variabilité spatio-temporelle des ressources) et l'intervention déterminante de scientifiques pour former et encadrer chaque participant aux méthodes préconisées.

Concernant la méthode d'enquête via les écoliers, une attention particulière a été portée aux choix des classes. 
En effet, il s'agit d'éviter les classes d'élèves trop âgées, où toutes les catégories sociales de la population ne sont pas représentées, en particulier les moins aisées, et qui ne garantiraient pas la représentativité des ménages enquêtés (Hosch, 2000). En Polynésie française, l'école étant obligatoire jusqu'à 16 ans, il est possible de travailler avec des classes dont la moyenne d'âge est plus élevée (au collège notamment, s'il en existe sur le site). En revanche, à Tuléar, les enfants qui ont participé sont des écoliers d'écoles primaires. En effet, les enfants quittant l'école relativement tôt (Ranaivomanana, 2006), aux environs de 12 ans, il est nécessaire de cibler des observateurs relativement jeunes afin de garantir la représentativité de l'échantillon des ménages enquêtés. Par ailleurs, quel que soit le site, il s'est avéré primordial de bénéficier de l'appui du chef d'établissement et des professeurs afin de renforcer l'intérêt des élèves et leur application à compléter le questionnaire une fois à la maison. Les indicateurs de suivi de la pêcherie renseignés sont nombreux et concernent l'activité de pêche, les menaces et la consommation en poissons.

Quelle que soit la méthode, à Tuléar, contrairement à la Polynésie française, les participants (mis à part les écoliers) ont reçu une compensation financière en fonction de leur contribution au programme de suivi. Cela s'est avéré nécessaire en raison du manque de sensibilité à la problématique de la gestion durable des ressources naturelles (Ranaivomanana, 2006) et du fait de la situation de pauvreté (Iida, 2005). Cette rémunération, qui reflète aussi le manque d'intérêt de la communauté pour ces activités de collecte de données, pose le problème de la fiabilité et de la pérennité du système d'information lié au suivi participatif et peut même se révéler contre-productive. Lorsque les fonds pour l'appui à ce type d'activité ne sont plus disponibles, alors le suivi n'est pas prolongé. Et pourtant, la mise en place de mesures incitatives s'avère très souvent indispensable au bon fonctionnement de tout programme de suivi participatif (Almany et al., 2010).

\section{Degré d'implication des participants}

La date, la durée, la technique utilisée, le site de pêche, la composition des captures et la taille des poissons capturés furent enregistrés par neuf pêcheurs volontaires à Moorea et dix à Tikehau via des cahiers de pêche, nombres insuffisants pour que des indicateurs relatifs à l'activité de pêche puissent être calculés. En outre, l'assiduité de ces pêcheurs fut faible, malgré un encadrement par des scientifiques pendant une année (9\% des sorties ont fait l'objet de relevés à Moorea, $8 \%$ à Tikehau).

Trois mareyeuses volontaires ont été identifiées, une pour chaque quartier d'Ankiembe, le principal site de débarquement de la baie de Tuléar. Ces mareyeuses, présentes sur le site de débarquement dans le cadre de leur activité professionnelle, devaient consacrer quelques minutes supplémentaires pour échantillonner chaque jour, pendant six mois, le débarquement de trois pirogues minimum chacune. À l'issue de l'expérience, $98 \%$ des relevés requis ont été réalisés. Le nombre de pirogues échantillonnées s'élève à 1586 , soit 4,3\% des 36922 sorties de pêche comptabilisées à Ankiembe sur cette période.

Seuls deux moniteurs de plongée et deux pêcheurs ont accepté de réaliser des comptages visuels en plongée à Moorea, tandis que deux moniteurs de plongée et un pêcheur se sont portés volontaires à Tikehau. Ils ont souhaité avoir leur site de comptage à proximité $\mathrm{d}$ 'un de leurs sites de plongée ou de pêche, et réaliser un échantillonnage par mois sur trois transects maximum. Les suivis ont été poursuivis, encadrés par un scientifique, pendant une année afin de mesurer l'assiduité des participants. À l'issue de cette année d'expérience, seuls $20 \%$ des comptages prévus ont été réalisés par les volontaires à Moorea et $24 \%$ à Tikehau. Après sept mois à Moorea et neuf mois à Tikehau, plus aucun échantillonnage n'a été réalisé.

À Moorea, sur les 201 écoliers (âge moyen : 14 ans) des deux collèges de l'île qui ont participé au programme de suivi, 137 ont retourné un questionnaire correctement complété, soit un taux de retour de $68 \%$. Ainsi, 127 ménages furent enquêtés $(4,4 \%$ de l'ensemble des ménages de Moorea). À Tikehau, il n'existe qu'une école primaire. Grâce aux élèves d'une des classes de cette école (âge moyen : 9 ans), dont le taux de retour des questionnaires a été de $96 \%$, 24 ménages furent enquêtés, représentant $35,3 \%$ des ménages de Tikehau. À Tuléar, douze classes des huit écoles primaires (élèves âgés d'une dizaine d'années) réparties dans tous les quartiers et villages de pêcheurs de la baie de Tuléar ont participé à l'expérience. Le nombre de ménages enquêtés s'élève à 326 (soit 10,3\% des ménages des villages et quartiers de pêcheurs de la baie de Tuléar), soit un taux de retour de $86 \%$.

Les résultats en matière de participation (nombre et assiduité des participants) pour chaque méthode testée, sur chaque site d'étude, sont synthétisés dans le tableau 3. Les enquêtes de perception, enquêtes via les écoliers et suivis des débarquements par les mareyeuses, sont caractérisées par une forte participation contrairement aux méthodes de suivi in situ et de suivi des captures via les cahiers de pêche. Pour ces deux dernières méthodes, l'assiduité des quelques volontaires est faible : la collecte de données devient quasiment nulle après quelques mois.

\section{Intérêt des informations générées par les suivis participatifs pour l'évaluation écosystémique des pêcheries récifales}

En moyenne, 21 indicateurs concernant le socioécosystème de la pêcherie sont renseignés par site, principalement grâce à deux méthodes : les enquêtes de 
Tableau 3. Évaluation du degré de participation de la population dans la mise en œuvre des méthodes de suivi participatif à Moorea, Tikehau et Tuléar.

\begin{tabular}{|c|c|c|c|c|}
\hline Méthode & Mesure de la participation & Moorea & Tikehau & Tuléar \\
\hline Enquêtes de perception & Nombre de participants : & 31 & 18 & 70 \\
\hline \multirow[t]{2}{*}{ Enquêtes via les écoliers } & Nombre de participants: & 137 & 24 & 326 \\
\hline & Taux de retour des questionnaires : & $68 \%$ & $96 \%$ & $86 \%$ \\
\hline \multirow[t]{2}{*}{ Suivi des captures } & Nombre de participants : & 9 & 10 & 3 \\
\hline & Taux de retour des fiches: & $9 \%$ & $\begin{array}{l}8 \% \text { (plus de retour } \\
\text { après } 6 \text { mois) }\end{array}$ & $98 \%$ \\
\hline \multirow{2}{*}{$\begin{array}{l}\text { Comptages visuels en } \\
\text { plongée }\end{array}$} & Nombre de participants : & 4 & 3 & non expérimenté \\
\hline & Taux de retour des fiches: & $\begin{array}{l}20 \% \text { (plus de retour } \\
\text { après } 7 \text { mois) }\end{array}$ & $\begin{array}{l}24 \% \text { (plus de retour } \\
\text { après } 9 \text { mois) }\end{array}$ & sur ce site \\
\hline
\end{tabular}

perception et les enquêtes via les écoliers. Tous ne sont pas directement utilisables pour évaluer la durabilité biologique des pêcheries en l'absence de valeurs de référence ou de séries temporelles de données qui permettraient de détecter des tendances. Les principaux indicateurs renseignés grâce aux méthodes du protocole proposé sont compilés dans un tableau de bord et comparés à des valeurs ou tendances de référence disponibles (Tab. 4). D'ores et déjà exploitables, ils seront plus facilement interprétables avec les méthodes mises en œuvre à intervalles réguliers et des tendances dans les valeurs des indicateurs pourront être dégagées. Ces analyses fournissent pour chaque site des informations pertinentes concernant la durabilité biologique de la pêcherie en question. Pour les pêcheries de Moorea et Tuléar, les informations générées par les suivis participatifs penchent toutes en faveur d'une surexploitation des ressources. Ces informations pointent à la fois une dégradation des ressources (diminution de l'abondance et de la taille) et des habitats, un accroissement de l'effort de pêche (pour Tuléar), une diminution de la part des captures destinées à l'autoconsommation, un rendement de la pêche et une densité de pêcheurs supérieurs aux valeurs de référence seuils considérées comme soutenables (McClanahan et al., 2002 ; Newton et al., 2007). À Tikehau, les nombreuses informations obtenues dressent un constat plus mitigé. À l'échelle de l'atoll, la ressource ne semble pas surexploitée, à part peut-être pour certaines espèces facilement accessibles, ciblées par la pêche au fusil (Epinephelus polyphekadion et Naso brevirostris) ou la pêche collecte (langoustes, bénitiers) qui, sur la base des perceptions des usagers, montreraient des tendances à la dégradation.

\section{Discussion - Conclusion}

L'objectif général de l'étude consistait à évaluer la pertinence des approches participatives pour contribuer au suivi des pêcheries récifales. Cette étude montre qu'il est possible d'identifier des méthodes utiles pour un suivi écosystémique des pêcheries récifales et adaptées aux connaissances et compétences des participants volontaires. Quel que soit le site d'étude, des méthodes simples reposant sur les ressources disponibles localement ont permis de valoriser les connaissances empiriques et de collecter des données variées qui favorisent l'évaluation écosystémique de la pêcherie.

Les méthodes testées ne sont pas toutes aussi performantes en termes de degré d'implication des participants. Les enquêtes de perception et les enquêtes via les écoliers s'avèrent les plus efficaces ( 34 indicateurs ont pu être renseignés sur les trois sites grâce à la première méthode et 26 grâce à la seconde). Elles se caractérisent par le fait qu'elles requièrent la participation de la société civile de façon ponctuelle, dans une fenêtre de temps relativement étroite, et qu'elles font appel à un encadrement très serré des participants pendant la durée de l'enquête. En effet, le recueil des connaissances détenues par les usagers, dans le cadre des enquêtes de perception, se fait grâce à la présence d'une personne formée à ce type d'enquête. Pour la collecte des données par les écoliers au sein de leur ménage, cette activité est encadrée, et même rendue presque obligatoire car réalisée dans le cadre de travaux scolaires. Les méthodes qui nécessitent de maintenir un niveau de contribution élevé et requièrent une forte assiduité des participants, sur un temps relativement long (en l'occurrence douze mois pour les suivis in situ et les cahiers de pêche, et six mois pour le suivi des débarquements par les mareyeuses) ont montré moins de résultats probants. En effet, le suivi des activités par les cahiers de pêche et les suivis in situ des ressources exploitées par comptage visuel en plongée ne se sont pas montrés concluants en Polynésie française en raison d'un manque d'implication des pêcheurs (participation et assiduité faibles). Autrement dit, la seule perspective de contribuer à servir la collectivité ne s'est pas avérée suffisante pour garantir la disponibilité, l'attention et la rigueur des méthodes de collectes régulières de données, en particulier sur le long terme. Ainsi, pour la collecte de données continue, il semble nécessaire d'accorder une incitation financière même minime aux participants (comme ce fut le cas pour les mareyeuses à Tuléar), pour 
Tableau 4. Tableau de bord des indicateurs de la durabilité biologique des pêcheries de Moorea, Tikehau et Tuléar, calculés à partir des données collectées dans le cadre des suivis participatifs.

\begin{tabular}{|c|c|c|c|c|c|}
\hline Indicateur & 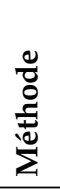 & 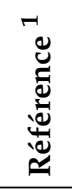 & Moorea & Tikehau & Tuléar \\
\hline Perception de l'évolution de l'abondance des poissons comestibles & $\mathrm{P}$ & $\downarrow$ & $\downarrow$ & $\downarrow^{5}$ & $\downarrow$ \\
\hline Perception de l'évolution de la taille des poissons comestibles & $\mathrm{P}$ & $\downarrow$ & $\downarrow$ & & $\downarrow$ \\
\hline Perception de l'évolution de la distance de fuite du poisson & $\mathrm{P}$ & $\uparrow$ & & & $\uparrow$ \\
\hline Perception de l'évolution de l'abondance des coquillages comestibles & $\mathrm{P}$ & $\downarrow$ & & & $\downarrow$ \\
\hline Perception de l'évolution de l'abondance des bénitiers & $\mathrm{P}$ & $\downarrow$ & $\downarrow$ & $\downarrow$ & \\
\hline Perception de l'évolution de l'abondance des langoustes & $\mathrm{P}$ & $\downarrow$ & & $\downarrow$ & $\downarrow$ \\
\hline Perception de l'évolution de l'abondance des crabes & $\mathrm{P}$ & $\downarrow$ & & & $\downarrow$ \\
\hline Perception de l'évolution de l'abondance des tortues & $\mathrm{P}$ & $\downarrow$ & & & $\downarrow$ \\
\hline Perception de l'évolution de l'abondance des holothuries comestibles & $\mathrm{P}$ & $\downarrow$ & & & $\downarrow$ \\
\hline Perception de l'évolution de l'abondance des oursins comestibles & $\mathrm{P}$ & $\downarrow$ & & & $\downarrow$ \\
\hline Perception de l'évolution du recouvrement en corail vivant & $\mathrm{P}$ & $\downarrow$ & $\downarrow$ & $\downarrow^{6}$ & $\downarrow$ \\
\hline Perception de l'évolution du recouvrement en macro-algues & $\mathrm{P}$ & $\uparrow$ & $\uparrow$ & $\uparrow$ & \\
\hline Perception de l'évolution du recouvrement des herbiers & $\mathrm{P}$ & $\downarrow$ & & & $\downarrow$ \\
\hline Perception de l'évolution du recouvrement des mangroves & $\mathrm{P}$ & $\downarrow$ & & & $\downarrow$ \\
\hline Perception de l'évolution de l'effort de pêche & $\mathrm{P}$ & $\uparrow$ & & $\downarrow$ & $\uparrow$ \\
\hline Perception de l'évolution de l'éloignement des zones de pêche & $\mathrm{P}$ & $\uparrow$ & & & $\uparrow$ \\
\hline Perception de l'évolution de la durée des sorties de pêche & $\mathrm{P}$ & $\uparrow$ & & & $\uparrow$ \\
\hline Perception de l'évolution de la fréquence des sorties de pêche & $\mathrm{P}$ & $\uparrow$ & & & $\uparrow$ \\
\hline Perception de l'évolution de la taille des mailles des filets & $\mathrm{P}$ & $\downarrow$ & & & $\uparrow$ \\
\hline Perception de l'évolution de la part des captures gardée pour autoconsommation & $\mathrm{P}$ & $\downarrow$ & & & $\downarrow$ \\
\hline Rendement de la pêche récifale $\left(\mathrm{t} \cdot \mathrm{an}^{-1} \cdot \mathrm{km}^{-2}\right)$ & $\mathrm{E}$ & $5^{2}$ & $24,5 \pm 6,0$ & $0,4 \pm 0,1$ & $14,2 \pm 2,3$ \\
\hline Nombre de pêcheurs par $\mathrm{km}^{2}$ & $\mathrm{E}$ & $5^{3}$ & $77^{4}$ & 0,2 & 10,4 \\
\hline Consommation en poissons récifaux $\left(\mathrm{kg} \cdot \mathrm{hab}^{-1} \cdot \mathrm{an}^{-1}\right)$ & $\mathrm{E}$ & $?$ & $83 \pm 21$ & $115 \pm 55$ & $50 \pm 4^{7}$ \\
\hline
\end{tabular}

${ }^{1}$ La référence correspond à la valeur ou la tendance de référence de l'indicateur. Tendance qui lorsqu'elle est suivie, ou valeur qui lorsqu'elle est dépassée, est potentiellement le signe d'une exploitation non durable. ${ }^{2}$ Newton et al., 2007. ${ }^{3}$ McClanahan et al., 2002. ${ }^{4} 64 \%$ étant des pêcheurs de loisir. ${ }^{5}$ Principalement Epinephelus polyphekadion et Naso brevirostris. ${ }^{6}$ Dans le lagon. ${ }^{7}$ Concerne uniquement les villages de pêcheurs de la baie de Tuléar. Pour chaque indicateur la méthode de collecte de données est précisée : enquête de perception (P) et enquête via les écoliers (E). $\downarrow$ : diminution. $\uparrow$ : augmentation.

s'assurer de leur persévérance et assiduité à compléter les fiches de relevé. Cela peut en partie s'expliquer par le fait que la gestion du milieu marin n'est pas ici déléguée à la communauté locale, comme c'est le cas pour les régimes fonciers coutumiers dans plusieurs îles du Pacifique par exemple. Or, une appropriation du milieu et une délégation de gestion renforcent l'intérêt, le sentiment de responsabilité et par conséquent l'engagement de la communauté envers la gestion de ce milieu (King, 2005). De plus, pour s'impliquer pleinement, les participants aux programmes de suivi doivent être persuadés que le temps qu'ils consacrent à la collecte de données est utile, c'està-dire que les données seront utilisées pour améliorer la situation (Stokes et al., 1990). Les informations produites doivent être correctement restituées, diffusées, discutées et intégrées au processus de prise de décision. Par ailleurs, le développement d'un plan de communication et un important travail d'animation sont essentiels pour accroître le nombre de participants et renforcer leur assiduité (Gouveia et al., 2004 ; Conrad et Daoust, 2008). Enfin, l'appropriation du suivi participatif par la communauté demande du temps et devrait se faire progressivement. En effet, face à une communauté où on se heurte à un manque de volonté de s'impliquer dans des programmes de collecte de données, il semble préférable $\mathrm{d}$ 'initier cette activité à très petite échelle et de l'associer très fortement à la mise en évidence des bénéfices d'une mesure de gestion. Par exemple, en 1997, à Fidji, la communauté de Ucunivanua a mesuré les conséquences de la fermeture d'une zone de 24 ha à l'exploitation d'une espèce de coquillage (Anadara sp.). Après deux années de fermeture, la communauté a pu observer que la densité en 
Anadara sp. avait augmenté de $1365 \%$ à l'intérieur de la zone protégée et de $523 \%$ à la périphérie. La mise en évidence de ce succès a incité la communauté du village en question et celles des villages voisins à mettre en place d'autres aires protégées (Johannes, 2002). Renforcer la participation des acteurs locaux aux activités de suivi et de gestion de l'environnement est un processus de longue haleine, il faut du temps et des compétences bien spécifiques pour acquérir la confiance de la population (Reed, 2008). Cela doit passer notamment par l'implication de celle-ci en amont de la phase de collecte de données et par des démonstrations à petite échelle de l'intérêt des activités de suivi. Ces éléments n'ayant pas été pris en compte dans le cadre de la présente recherche, cela peut expliquer en partie la faible implication des usagers dans certaines méthodes de suivi participatif testées. En effet, ces méthodes ont été mises en œuvre sur les sites d'étude dans le cadre d'une expérimentation scientifique, dont les objectifs étaient d'analyser leur capacité à générer des informations pertinentes pour l'évaluation des pêcheries récifales. Alors que pour être pleinement efficace, un programme de suivi participatif devrait s'inscrire dans une démarche globale de gestion participative des ressources, dans laquelle les acteurs et usagers locaux sont associés et les résultats des suivis incorporés au processus de prise de décision.

Il ressort de cette expérience que les approches participatives testées se révèlent peu appropriées pour des suivis continus, réguliers et à long terme de l'état de l'environnement, dans des pays où le contexte socioéconomique et culturel est tel que la société civile est peu encline à s'investir dans ce type de programme. En revanche, ces approches participatives montrent un réel potentiel pour des évaluations ponctuelles et à grande échelle du milieu étudié à condition que les participants soient encadrés (ex.: obligations scolaires, incitations financières, etc.). La concentration des phases de collecte de données dans le temps a le double avantage de n'éroder ni les budgets ni l'assiduité des participants. L'effort à déployer pour mobiliser des volontaires sur le court terme étant plus raisonnable, cette approche semble plus réaliste que des suivis continus. En effet, en profitant des connaissances et de l'expertise disponibles localement, il est possible d'obtenir en peu de temps un très grand nombre d'informations variées, de qualité et pertinentes pour caractériser et établir un diagnostic sur l'état d'un écosystème exploité, comme les expériences menées dans le cadre de cette étude le prouvent.

Cependant, ces approches peuvent se heurter à un certain nombre de difficultés. En tout premier lieu, la disponibilité des volontaires est un facteur limitant de taille. Dans certains cas, en particulier pour les pays en voie de développement, il peut s'avérer extrêmement difficile de trouver des volontaires prêts à donner de leur temps et de leur énergie pour contribuer au suivi de l'environnement. Dans ces pays, les membres des communautés locales vivent souvent dans des conditions difficiles. Bien que conscients des pressions qui pèsent sur le milieu qu'ils exploitent, leur situation de survie les contraint à ne pas s'impliquer dans des activités autres que celles qui leur permettent de subvenir aux besoins élémentaires de leurs familles. Leur vision est à court terme et répond à des exigences de survie au jour le jour. Ainsi, il est très difficile de les impliquer dans des activités bénévoles de suivi de l'état de l'environnement qui contribuent à une gestion durable des ressources, notion qui repose sur une vision à long terme. Parfois, pour des raisons culturelles, les communautés ne partagent pas les préoccupations environnementales qui nourrissent la motivation des volontaires à s'impliquer dans des programmes de suivi de l'environnement. C'est le cas par exemple des Vezo de Tuléar, une communauté de pêcheurs pour qui la disponibilité en ressources est conditionnée par des éléments divins. La Polynésie française où la sensibilité aux problématiques environnementales n'est pas aussi développée qu'en Occident en est un autre exemple. Dans ces conditions, il devient très difficile de bâtir et maintenir à long terme un programme de suivi participatif de l'environnement. Dans les pays où la conscience écologique de la société civile est forte, il est plus aisé d'impliquer des volontaires dans ces activités de suivi. C'est le cas en Australie, en Europe ou en Amérique du Nord, où les programmes de suivi participatifs foisonnent (Harvey et al., 2001 ; Whitelaw et al., 2003 ; Goffredo et al., 2004 ; Schmeller et al., 2009).

Une seconde difficulté concerne la nature des données obtenues. Tout d'abord, un grand nombre de données sont souvent d'ordre qualitatif. Or, celles-ci ne se prêtent pas à des analyses statistiques paramétriques puissantes permettant de tester des tendances ou des relations entre les phénomènes observés et les activités humaines. Cela n'enlève cependant rien à la pertinence de l'ensemble des informations pour fournir une vision cohérente et informative, et contribuer à une approche écosystémique de la pêcherie. Par ailleurs, les informations apportées par les suivis participatifs peuvent plus facilement faire l'objet de contestations, car elles sont collectées par des nonprofessionnels (bien que les professionnels ne soient pas à l'abri de ce type de difficulté) qui peuvent être à l'origine de biais lorsque l'encadrement par les scientifiques n'est pas suffisant (Léopold et al., 2009). En cas de conflit, il peut être difficile pour les gestionnaires de s'appuyer sur ces données pour justifier auprès des politiques ou de la population la mise en place d'une ou plusieurs mesures de gestion ; le suivi participatif devrait cependant favoriser la communication et la concertation entre les acteurs concernés.

Une autre difficulté encore porte sur l'effort à déployer afin d'assurer l'animation et la coordination d'un programme de suivi participatif qui peut être 
rédhibitoire, notamment lorsque cet effort doit être maintenu sur le long terme. L'expérience montre en effet qu'il est nécessaire de déployer d'importants efforts en matière de communication, animation, motivation, compensation et valorisation des volontaires, supervision scientifique (établissement du protocole de collecte de données, formation, vérification de la qualité des données, analyse des données) et restitution des informations. Suivi participatif ne signifie pas absence d'implication des scientifiques, bien au contraire : des moyens et des compétences en animation et en communication doivent compléter le dispositif. Dans le cadre de suivis continus, c'est-à-dire impliquant la collecte de données à une fréquence élevée et sur le long terme, ces efforts d'encadrement doivent être constants. Tout relâchement se traduit rapidement par une réduction puis un abandon des activités de collecte de données par les participants. C'est pourquoi une cellule de coordination est généralement nécessaire, coordination entre scientifiques, personnes en charge du suivi et volontaires. Ainsi, les coûts associés à la coordination des suivis participatifs par les scientifiques, ainsi que ceux liés au fonctionnement de la structure d'animation ne sont pas négligeables.

Mais analyser la pertinence des suivis participatifs uniquement selon des critères de faisabilité et de mise à disposition de données est insuffisant. Un programme de suivi participatif a d'autres intérêts que celui de produire des données nombreuses et pertinentes pour le suivi des pêcheries récifales. En matière de gestion, pour être réellement efficaces, les données collectées dans le cadre d'un suivi doivent être compréhensibles et appropriées localement, c'est-à-dire qu'elles doivent répondre à de réels besoins en information, être diffusées auprès de la population et surtout être utilisées et se concrétiser sous la forme de décisions mises en œuvre (Johannes, 1998 ; Sheil, 2001 ; Nichols et Williams, 2006). Sinon, l'effort aura été vain. Or il a été démontré qu'un des avantages des suivis participatifs est de faciliter le lien entre la production d'information et son utilisation par les instances de décision locales (Danielsen et al., 2005b ; Danielsen et al., 2007 ; Danielsen et al., 2010), à condition de répondre localement à un besoin. L'implication par exemple de volontaires dans les suivis de type Global Coral Reef Monitoring Network (GCRMN), qui répondent au départ à un besoin international (évaluation de l'état mondial des récifs coralliens), n'est probante que si les données ont parallèlement une pertinence au niveau local. Un deuxième avantage concerne la sensibilisation et l'éducation de la société civile à la préservation de l'environnement (Aswani et Weiant, 2004) : le changement de comportement des usagers est la clé pour assurer la pérennité des écosystèmes coralliens sur le long terme. En effet, les actions de sensibilisation permettent d'agir en profondeur sur la perception et les relations que la population entretient avec l'environnement et de favoriser un plus grand respect du milieu marin. En particulier, la méthode des enquêtes via les écoliers présente l'avantage de contribuer à l'éducation environnementale de la jeune génération. La littérature souligne également le potentiel des suivis participatifs à contribuer au renforcement des capacités des acteurs du socio-écosystème à détecter les changements de l'écosystème et à pouvoir s'y adapter, tout en favorisant leur participation aux processus de gestion (Danielsen et al., 2005a ; Uychiaoco et al., 2005; Almany et al., 2010). Cependant, pour que ces propriétés puissent s'exprimer, le suivi participatif doit s'insérer dans un cadre de gouvernance plus large, favorisant la participation des acteurs et usagers locaux, tel que le cadre de la gestion intégrée de la zone côtière (David et al., 2010).

En conclusion, la présente recherche a permis de démontrer l'utilité des approches participatives pour générer ponctuellement des informations pertinentes pour le suivi des pêcheries récifales. Les suivis participatifs présentent un potentiel pour renforcer l'intérêt, l'appropriation et la participation active et efficace des populations locales dans les processus de gestion. Ce potentiel mériterait d'être analysé pour conforter la démonstration de la pertinence de ces suivis en tant qu' outils contribuant à une gestion durable des pêcheries récifales.

\section{Remerciements}

Cette recherche a été menée dans le cadre d'une thèse de doctorat financée par une bourse de formation à la recherche de l'Agence universitaire de la francophonie. Elle a également bénéficié du soutien financier de InitiativeS Corail pour le Pacifique (Crisp), du service de la Pêche de Polynésie française, de la Fondation Naturalia et Biologia, et de l'Action thématique interdépartementale sur les aires protégées de l'Institut de recherche pour le développement. Sur le terrain, l'aide de Nicolas Maihota en Polynésie française et de Jo Massina à Madagascar fut très précieuse.

\section{Références}

Almany, G.R., Hamilton, R.J., Williamson, D.H., Evans, R.D., Jones, G.P., Matawai, M., Potuku, T., Rhodes, K.L., Russ, G.R., Sawynok, B., 2010. Research partnerships with local communities: Two case studies from Papua New Guinea and Australia, Coral Reefs, 29, 567-576.

Andrianandrasana, H. T., Durbin, J., Lewis, R.E, Randriamahefasoa, J., Ratsimbazafy, J.H., 2005. Participatory ecological monitoring of the Alaotra wetlands in Madagascar, Biodiversity and Conservation, 14, 11, 2757-2774. 
Aswani, S., Weiant, P., 2004. Scientific evaluation in women's participatory management: Monitoring marine invertebrate refugia in the Solomon Islands, Human Organization, 63, 3, 301-319.

Brenier, A., Ferraris, J., Galzin, R., 2007. Community-based monitoring of coral reef fish resources in two contrasted islands of French Polynesia. $21^{\text {st }}$ Pacific Science Congress, Okinawa, Japan, 12-18 June.

Brenier, A., Mahafina, J., Ranaivoson, E., Ferraris, J., 2009a. Participatory assessment of ecological sustainability of the Toliara Bay reef fishery. Sixth Western Indian Ocean Marine Science Association Scientific Symposium, Saint-Denis, Université de La Réunion, France, 24-29 August.

Brenier, A., Mahafina, J., Galzin, R., Ferraris, J., 2009b. Relevance of participatory approaches for ecosystemic monitoring of reef fisheries. Second International Marine Protected Areas Conference, Washington D.C, 19-24 May.

Bunce, M., Rodwell, L.D., Gibb, R., Mee, L., 2008. Shifting baselines in fishers' perceptions of island reef fishery degradation, Ocean and Coastal Management, 51, 4, 285-302.

Caddy, J.F., 2004. Current usage of fisheries indicators and reference points, and their potential application to management of fisheries for marine invertebrates, Canadian Journal of Fisheries and Aquatic Sciences, 61, 1307-1324.

Clua, E., Beliaeff, B., Chauvet, C., David, G., Ferraris, J., Kronen, M., Kulbicki, M., Labrosse, P., Letourneur, Y., Pelletier, D., Thebaud, O., Leopold, M., 2005. Towards multidisciplinary indicator dashboards for coral reef fisheries management, Aquatic Living Resources, 18, 3, 199-213.

Connell, J.H., 1978. Diversity in tropical rain forest and coral reefs: High diversity of trees and corals is maintained only in a non-equilibrium state, Science, 199, 4335, 1302-1310.

Conrad, C.T., Daoust, T., 2008. Community-based monitoring frameworks: Increasing the effectiveness of environmental stewardship, Environmental Management, 41, 3, 358-366.

Dalzell, P., Adams, T.J.H., Polunin, N.V.C., 1996. Coastal fisheries in the Pacific islands, Oceanography and Marine Biology: An Annual Review, 34, 395-531.

Danielsen, F., Burgess, N. D., Balmford, A., 2005a. Monitoring matters: Examining the potential of locally-based approaches, Biodiversity and Conservation, 14, 11, 2507-2542.

Danielsen, F., Jensen, A.E., Alviola, P.A., Balete, D.S., Mendoza, M., Tagtag, A., Custodio, C., Enghoff, M., 2005b. Does monitoring matter? A quantitative assessment of management decisions from locally-based monitoring of protected areas, Biodiversity and Conservation, 14, 11, 26332652.

Danielsen, F., Mendoza, M.M., Tagtag, A., Alviola, P.A., Balete, D.S., Jensen, A.E., Enghoff, M., Poulsen, M.K., 2007. Increasing conservation management action by involving local people in natural resource monitoring, Ambio, 36, 7, 566-570.

Danielsen, F., Burgess, N.D., Jensen, P.M., Pirhofer-Walzl, K., 2010. Environmental monitoring: The scale and speed of implementation varies according to the degree of people's involvement, Journal of Applied Ecology, 47, 1166-1168.

Darwall, W.R.T., Dulvy, N.K., 1996. An evaluation of the suitability of non-specialist volunteer researchers for coral reef fish surveys. Mafia Island, Tanzania: A case study, Biological Conservation, 78, 3, 223-231.

David, G., Leopold, M., Dumas, P.S., Ferraris, J., Herrenschmidt, J.B., Fontenelle, G., 2010. Integrated coastal zone management perspectives to ensure the sustainability of coral reefs in New Caledonia, Marine Pollution Bulletin, 61, 323-334.

Debrot, A.O., Nagelkerken, I., 2000. User perceptions on coastal resource state and management options in Curacao, Revista de Biologia Tropical, 48, 95-106.

Delaney, D.G., Sperling, C.D., Adams, C.S., Leung, B., 2008. Marine invasive species: Validation of citizen science and implications for national monitoring networks, Biological Invasions, 10, 1, 117-128.

FAO, 2003. The ecosystem approach to fisheries, Technical Guidelines for Responsible Fisheries, 4, 2, FAO, Rome.

Ferraris, J., Cayré, P., 2003. Les pêcheries récifales dans le Pacifique Sud : d'une gestion intuitive vers une gestion écosystémique raisonnée, Océanis, 29, 3-4, 397-414.

Garcia, S.M., Zerbi, A., Aliaume, C., Do Chi, T., Lasserre, G., 2003. The ecosystem approach to fisheries: Issues, terminology, principles, institutional foundations, implementation and outlook, FAO FisheriesTechnical Paper, 443, FAO, Rome.

Goffredo, S., Piccinetti, C., Zaccanti, F., 2004. Volunteers in marine conservation monitoring: A study of the distribution of seahorses carried out in collaboration with recreational scuba divers, Conservation Biology, 18, 6, 1492-1503.

Goffredo, S., Pensa, F., Neri, P., Orlandi, A., Gagliardi, M.S., Velardi, A., Piccinetti, C., Zaccanti, F., 2010. Unite research with what citizens do for fun: "Recreational monitoring" of marine biodiversity, Ecological Applications, 20, 2170-2187.

Gouveia, C., Fonseca, A., Camara, A., Ferreira, F., 2004. Promoting the use of environmental data collected by concerned citizens through information and communication technologies, Journal of Environmental Management, 71, 2, 135-154.

Harvey, N., Clarke, B.D., Carvalho, P., 2001. The role of the Australian Coastcare program in community-based coastal management: A case study from South Australia, Ocean $\mathcal{E}$ Coastal Management, 44, 3-4, 161-181.

Hilborn, R., 2007. Moving to sustainability by learning from successful fisheries, Ambio, 36, 4, 296-303.

Hodgson, G., 1999. A global assessment of human effects on coral reefs, Marine Pollution Bulletin, 38, 5, 345-355.

Hosch, G., 2000. The use of students in surveying subsistence fisheries: A Pacific island case study, FAO Fisheries Circular, 962, FAO, Rome.

Iida, T., 2005. The past and present of the coral reef fishing economy in Madagascar: Implication for selfdetermination in resource use, Senri Ethnological Studies, 67, 237-258.

Jackson, J.B.C., Kirby, M.X., Berger, W.H., Bjorndal, K.A., Botsford, L.W., Bourque, B.J., Bradbury, R.H., Cooke, R., Erlandson, J., Estes, J.A., Hughes, T.P., Kidwell, S., Lange, C. B., Lenihan, H.S., Pandolfi, J.M., Peterson, C.H., Steneck, R.S., Tegner, M.J., Warner, R.R., 2001. Historical overfishing and the recent collapse of coastal ecosystems, Science, 293, 5530, 629-638.

Jackson, J.B.C., 2007. Economic incentives, social norms, and the crisis of fisheries, Ecological Research, 22, 1, 16-18. 
Jennings, S., Polunin, N.V.C., 1995. Comparative size and composition of yield from six Fijian reef fisheries, Journal of Fish Biology, 46, 1, 28-46.

Jennings, S., Polunin, N.V C., 1996. Impacts of fishing on tropical reef ecosystems, Ambio, 25, 1, 44-49.

Jennings, S., 2005. Indicators to support an ecosystem approach to fisheries, Fish and Fisheries, 6, 3, 212-232.

Johannes, R.E., 1998. The case for data-less marine resource management: Examples from tropical nearshore finfisheries, Trends in Ecology E Evolution, 13, 6, 243-246.

Johannes, R.E., 2002. The renaissance of community-based marine resource management in Oceania, Annual Review of Ecology and Systematics, 33, 317-340.

King, M., 2005. Problems with centralised fisheries management in Pacific islands, in Kishigami, N., Savelle, J.M. (Eds), Indigenous Use and Management of Marine Resources, Osaka, Japan, National Museum of Ethnology, 181-195.

Léopold, M., Cakacaka, A., Meo, S., Sikolia, J., Lecchini, D., 2009. Evaluation of the effectiveness of three underwater reef fish monitoring methods in Fiji, Biodiversity and Conservation, 18, 3367-3382.

Lunn, K. E., Dearden, P., 2006. Monitoring small-scale marine fisheries: An example from Thailand's Ko Chang archipelago, Fisheries Research, 77, 1, 60-71.

McClanahan, T., Polunin, N., Done, T., 2002. Ecological states and the resilience of coral reefs, Conservation Ecology, 6, 2, 18 .

Mumby, P.J., Dahlgren, C.P., Harborne, A.R., Kappel, C.V., Micheli, F., Brumbaugh, D.R., Holmes, K.E., Mendes, J.M., Broad, K., Sanchirico, J.N., Buch, K., Box, S., Stoffle, R.W., Gill, A.B., 2006. Fishing, trophic cascades, and the process of grazing on coral reefs, Science, 311, 5757, 98-101.

Newman, C., Buesching, C.D., Macdonald, D.W., 2003. Validating mammal monitoring methods and assessing the performance of volunteers in wildlife conservation: "Sed quis custodiet ipsos custodies?", Biological Conservation, 113, 2, 189-197.

Newton, K., Cote, I.M., Pilling, G.M., Jennings, S., Dulvy, N.K., 2007. Current and future sustainability of island coral reef fisheries, Current Biology, 17, 7, 655-658.

Nichols, J.D., Williams, B.K., 2006. Monitoring for conservation, Trends in Ecology \& Evolution, 21, 12, 668-673.

Obura, D.O., 2001. Participatory monitoring of shallow tropical marine fisheries by artisanal fishers in Diani, Kenya, Bulletin of Marine Science, 69, 2, 777-791.

Obura, D.O., Wells, S., Church, J., Horrill, C., 2002. Monitoring of fish and fish catches by local fishermen in Kenya and Tanzania, Marine and Freshwater Research, 53, 2, 215-222.

Pattengill-Semmens, C.V., Semmens, B.X., 2003. Conservation and management applications of the reef volunteer fish monitoring program, Environmental Monitoring and Assessment, 81, 1-3, 43-50.

Pikitch, E.K., Santora, C., Babcock, E.A., Bakun, A., Bonfil, R., Conover, D.O., Dayton, P., Doukakis, P., Fluharty, D., Heneman, B., Houde, E.D., Link, J., Livingston, P.A., Mangel, M., McAllister, M.K., Pope, J., Sainsbury, K.J., 2004. Ecosystem-based fishery management, Science, 305, 5682, 346-347.

Ranaivomanana, L.N.J., 2006. Identification des conditions d'appropriation de la gestion durable des ressources naturelles et des écosystèmes : "Cas du Grand Récif de Toliara ". Thèse de doctorat, Université de Toliara, Tuléar, Madagascar.

Reed, M.S., 2008. Stakeholder participation for environmental management: A literature review, Biological Conservation, 141, 2417-2431.

Rist, J., Milner-Gulland, E. J., Cowlishaw, G., Rowcliffe, M., 2010. Hunter reporting of catch per unit effort as a monitoring tool in a bushmeat-harvesting system, Conservation Biology, 24, 489-499.

Rochet, M.J., Trenkel, V. M., 2003. Which community indicators can measure the impact of fishing? A review and proposals, Canadian Journal of Fisheries and Aquatic Sciences, 60, 86-99.

Rochet, M.J., Prigent, M., Bertrand, J.A., Carpentier, A., Coppin, F., Delpech, J.P., Fontenelle, G., Foucher, E., Mahe, K., Rostiaux, E., Trenkel, V.M., 2008. Ecosystem trends: Evidence for agreement between fishers' perceptions and scientific information, Ices Journal of Marine Science, 65, 1057-1068.

Russ, G.R., 1991. Coral reef fisheries: Effects and yields, in Sale, P.F. (Ed.), The Ecology of Fishes on Coral Reefs, San Diego, Academic Press, 601-635.

Savan, B., Morgan, A J., Gore, C., 2003. Volunteer environmental monitoring and the role of the universities: The case of Citizens' Environment Watch, Environmental Management, 31, 5, 561-568.

Schmeller, D.S., Henry, P.Y., Julliard, R., Gruber, B., Clobert, J., Dziock, F., Lengyel, S., Nowicki, P., Deri, E., Budrys, E., Kull, T., Tali, K., Bauch, B., Settele, J., Van Swaay, C., Kobler, A., Babij, V., Papastergiadou, E., Henle, K., 2009. Advantages of volunteer-based biodiversity monitoring in Europe, Conservation Biology, 23, 2, 307-316.

Sheil, D., 2001. Conservation and biodiversity monitoring in the tropics: Realities, priorities, and distractions, Conservation Biology, 15, 4, 1179-1182.

Stokes, P.,Havas, M., Brydges, T., 1990. Public-participation and volunteer help in monitoring programs: An assessment, Environmental Monitoring and Assessment, 15, 3, 225-229.

Tawake, A., Parks, J., Radikedike, P., Aalbersberg, B., Vuki, V., Salafsky, N., 2001. Harvesting clams and data involving local communities in monitoring can lead to conservation success in all sorts of unanticipated ways: A case in Fiji, Conservation in Practice, 2, 4, 32-35.

Teh, L., Cabanban, A.S., Sumaila, U.R., 2005. The reef fisheries of Pulau Banggi, Sabah: A preliminary profile and assessment of ecological and socio-economic sustainability, Fisheries Research, 76, 3, 359-367.

Teh, L., Sumaila, U. R., 2007. Malthusian overfishing in Pulau Banggi?, Marine Policy 31, 4, 451-457.

Trenkel, V.M., Rochet, M.J., Mesnil, B., 2007. From modelbased prescriptive advice to indicator-based interactive advice, Ices Journal of Marine Science, 64, 4, 768-774.

Uychiaoco, A.J., Arceo, H.O., Green, S.J., De la Cruz, M.T., Gaite, P.A., Alino, P.M., 2005. Monitoring and evaluation of reef protected areas by local fishers in the Philippines: Tightening the adaptive management cycle, Biodiversity and Conservation, 14, 11, 2775-2794. 
Walmsley, S.F., White, A.T., 2003. Influence of social, management and enforcement factors on the long-term ecological effects of marine sanctuaries, Environmental Conservation, 30, 4, 388-407.

Webb, E.L., Maliao, R.J., Siar, S.V., 2004. Using local user perceptions to evaluate outcomes of protected area management in the Sagay Marine Reserve, Philippines, Environmental Conservation, 31, 2, 138-148.

Whitelaw, G., Vaughan, H., Craig, B., Atkinson, D., 2003. Establishing the Canadian community monitoring

Reçu le 16 décembre 2008. Accepté le 12 septembre 2012 network, Environmental Monitoring and Assessment, 88, 1-3, 409-418.

Wiber, M., Berkes, F., Charles, A., Kearney, J., 2004. Participatory research supporting community-based fishery management, Marine Policy, 28, 6, 459-468.

Wilkinson, C., 2004. Status of Coral Reefs of the World: 2004. Global Coral Reef Monitoring Network, Australian Institute of Marine Science, Townsville, Australia.

Zeller, D., Booth, S., Craig, P., Pauly, D., 2006. Reconstruction of coral reef fisheries catches in American Samoa, 19502002, Coral Reefs, 25, 1, 144-152. 